\title{
Hyperthermic Intraperitoneal Chemotherapy in Advanced Gastric Cancer: The End of Skepticism?
}

\author{
Olivier Glehen, MD, PhD, François Noël Gilly, MD, PhD, and Eddy Cotte, MD \\ Surgical Oncology Department, Centre Hospitalo-Universitaire Lyon Sud, Pierre Bénite, France
}

Over the past two decades, novel therapeutic approaches to peritoneal carcinomatosis (PC) have emerged, combining cytoreductive surgery and peritonectomy procedures with perioperative intraperitoneal chemotherapy (PIC), including hyperthermic intraperitoneal chemotherapy (HIPEC) and/or early postoperative intraperitoneal chemotherapy (EPIC). Theoretically, cytoreductive surgery is performed to treat macroscopic disease and PIC to treat microscopic residual disease aiming to remove disease completely with a single procedure. Many consider it a standard of care for disease such as pseudomyxoma peritonei, peritoneal mesothelioma, or localized and resectable colorectal carcinomatosis. ${ }^{1}$ Because of its negative prognosis, the question regarding the efficiency of this combined procedure still remains controversial for carcinomatosis from gastric origin.

Peritoneal dissemination is the most frequent pattern of metastasis and recurrence with gastric cancers. Traditionally, there was a mutual agreement in the oncologic community that those patients with gastric peritoneal dissemination were incurable. For the past 10 years, despite improvements in systemic chemotherapy with the development of new targeted therapy and encouraging tumor response rates, there have been no large phase III studies that demonstrated the real benefit of one regimen and really changed the negative prognosis of this disease evolution. ${ }^{2}$ With this phase III study including 68 patients with PC from gastric cancer, Yang et al. ${ }^{3}$ demonstrated that the use of HIPEC with the association cisplatinum-mitomycin $\mathrm{C}$ significantly improved survival. With HIPEC, the overall survival was extended by nearly $70 \%$ (6.5 vs 11.0 months) when compared with cytoreductive surgery alone. The

(C) Society of Surgical Oncology 2011

Published Online: 8 March 2011

O. Glehen, $\mathrm{MD}, \mathrm{PhD}$

e-mail: olivier.glehen@chu-lyon.fr authors compared their results and the design of their study to those reported by the Netherlands phase III study, conducted for the treatment of PC from colorectal origin and that compared the combination of CRS with HIPEC compared with systemic chemotherapy. ${ }^{4}$ This important study strongly influenced the oncologic community and precipitated the evolution to a new management of colorectal carcinomatosis with a curative intent. However, it demonstrated the potential benefit of CRS combined with HIPEC and not of HIPEC only. To our knowledge, Yang et al. ${ }^{3}$ is the first study that demonstrated the benefit of HIPEC in one phase III study for the treatment of PC, whatever the origin is.

The experience of few single institutions, combined with phase II studies, reported encouraging survival results following treatment of PC with this therapeutic strategy. ${ }^{5-7}$ Recently, a collaborative effort of French institutions collected data from 159 patients and represents the largest experience of the treatment of PC from gastric origin. ${ }^{8}$ With a median follow-up of 20.4 months, the overall median survival was 9.2 months, which is close to that reported by Yang et al. ${ }^{3}$ in the experimental group (11 months). This median survival rate remains low, but the 5-year survival rate was $13 \%$ with some long-term survivors. These survival results are less encouraging than those obtained for other peritoneal surface malignancies, reflecting either a more aggressive disease process less responsive to this combined treatment modality or the need for better patient selection. However, the combination of cytoreductive surgery with HIPEC was the only therapeutic strategy that reported long-term survivors at 5 years. ${ }^{6,7,9}$

Prognostic factors are essential for a better patient selection for the combined procedure, and some have been identified in the phase III study. For intraperitoneal chemotherapy to be effective, residual disease following attempted cytoreductive surgery must be of low volume. In the recent French study, long-term survival, with a 5-year 
survival of $23 \%$ and a median survival of 15 months, was obtained in patients treated with complete macroscopic resection. ${ }^{8}$ Yonemura et al. reported similar encouraging results with a 5-year survival rate of $27 \%$ and a median survival of 15.5 months for patients with gastric PC treated with complete cytoreduction and HIPEC. ${ }^{5}$ Hall et al. observed a median survival of 11.2 months for CC-0 patients with gastric PC treated with HIPEC using mitomycin $\mathrm{C}$, whereas no patient with residual disease was alive after 2 years. ${ }^{10}$ An aggressive attempt at complete resection, including surgical excision of all sites of macroscopic disease, may add to the efficacy of HIPEC. When cytoreductive surgery does not allow sufficient downstaging, the survival benefit of HIPEC remains extremely low, and median survival did not exceed 8 months. In light of the risk of postoperative complications, HIPEC should not be indicated in patients with residual tumor nodules.

Surprisingly, the carcinomatosis extent assessed by Sugarbaker's Peritoneal Cancer Index was not an independent prognostic factor in the Yang et al. ${ }^{3}$ study, whereas it demonstrated significant influence on survival in many other studies. $8,11,12$ PC with localized or small tumor nodules seems to be the best indication for this combined procedure. In patients who had undergone complete cytoreductive surgery in the French study, the extent of carcinomatosis represented the only strong prognostic factor. ${ }^{8}$ When the PCI was more than 12 , despite a complete cytoreductive surgery, no patient was alive at 3 years. Fujimoto et al. also reported impressive survival results in patients with limited PC (5-year survival rates of 40-50\%), whereas 1-year survival rate was only $18 \%$ for patients with more extensive PC. ${ }^{7}$ Regarding these results, it may not be recommended to propose this combined treatment for patients with extensive PC, even if complete cytoreductive surgery appears to be possible. Patients with gastric PC and Sugarbaker's PCI of more than 12 should be contraindicated for this kind of therapeutic strategy.

Since the publication of recent randomized trials, the use of neoadjuvant chemotherapy has been routinely used and recommended, especially in Europe for the management of gastric adenocarcinoma without PC. ${ }^{13}$ It may help physicians to better select indicators. Patients who progress or develop extra-abdominal metastases during this preoperative treatment may be excluded for an aggressive and complex procedure that would probably not improve prognosis because of postoperative and systemic progression of disease. The benefit of this perioperative systemic chemotherapy appears to also be suggested by the fact that the use of more than 6 cycles of chemotherapy was found to be a positive independent prognostic factor by the phase III study and may play a role by limiting the evolution of metastatic disease.
The institution in which the procedure was performed plays an important role for the postoperative course and for survival. It is, however, reasonable to make the assumption that experience may provide better patient selection, that is, surgical expertise with a higher rate of complete cytoreductive surgery and postoperative management. A learning curve has been reported by several authors and institutions performing the combination of cytoreductive surgery with HIPEC for the management of PC. ${ }^{14,15}$ All interventional complex procedures have an inherent risk, and experience undoubtedly diminishes this, but can never abolish it. In the Yang et al. ${ }^{3}$ study, HIPEC did not significantly enhance the rates of morbidity and mortality, although it was performed by a specialized team involved in the management of gastric and PC surgery. As the occurrence of serious adverse event appears to be an independent prognostic factor, adequate patient selection by the specialized team is probably essential.

Whereas HIPEC was largely and positively evaluated prophylactically or in an adjuvant setting in phase III studies by Korean and Japanese researchers, Yang et al. ${ }^{3}$ reported the first phase III study that evaluated this technique for established PC from gastric origin. ${ }^{16}$ It demonstrated that HIPEC should be considered an important therapeutic tool for the management of this disease. However, the survival results and the prognostic factors reported underline the necessity of strict selection of patients for such procedures.

\section{REFERENCES}

1. Glehen O, Gilly FN, Boutitie F, Bereder JM, Quenet F, Sideris L, et al. Toward curative treatment of peritoneal carcinomatosis from nonovarian origin by cytoreductive surgery combined with perioperative intraperitoneal chemotherapy: a multi-institutional study of 1,290 patients. Cancer. 2010;116:5608-18.

2. Boku N. Chemotherapy for metastatic disease: review from JCOG trials. Int J Clin Oncol. 2008;13:196-200.

3. Yang X-J, Huang C-Q, Suo T, Mei L-J, Yang G-L, Cheng F-L et al. Cytoreductive surgery and hyperthermic intraperitoneal chemotherapy improves survival of patients with peritoneal carcinomatosis from gastric cancer: final results of a phase III randomized clinical trial. Ann Surg Oncol. doi: 10.1245/ s10434-011-1631-5.

4. Verwaal VJ, Bruin S, Boot H, van Slooten G, van Tinteren H. 8-year follow-up of randomized trial: cytoreduction and hyperthermic intraperitoneal chemotherapy versus systemic chemotherapy in patients with peritoneal carcinomatosis of colorectal cancer. Ann Surg Oncol. 2008;15:2426-32.

5. Yonemura Y, Kawamura T, Bandou E, Takahashi S, Sawa T, Matsuki N. Treatment of peritoneal dissemination from gastric cancer by peritonectomy and chemohyperthermic peritoneal perfusion. Br J Surg. 2005;92:370-5.

6. Glehen O SV, Cotte E, Sayag-Beaujard AC, Osinsky D, Freyer G, Francois $\mathrm{Y}$, et al. Cytoreductive surgery and intraperitoneal chemohyperthermia for peritoneal carcinomatosis arising from gastric cancer. Arch Surg. 2004;139:20-6. 
7. Fujimoto S, Takahashi M, Mutou T, Kobayashi K, Toyosawa T, Isawa $\mathrm{E}$, et al. Improved mortality rate of gastric carcinoma patients with peritoneal carcinomatosis treated with intraperitoneal hyperthermic chemoperfusion combined with surgery. Cancer. 1997;79:884-891.

8. Glehen O, Gilly FN, Arvieux C, Cotte E, Boutitie F, Mansvelt B, et al. Peritoneal carcinomatosis from gastric cancer: a multiinstitutional study of 159 patients treated by cytoreductive surgery combined with perioperative intraperitoneal chemotherapy. Ann Surg Oncol. 2010;17:2370-7.

9. Yonemura Y, Fujimura T, Nishimura G, Falla R, Sawa T, Katayama K, et al. Effects of intraoperative chemohyperthermia in patients with gastric cancer with peritoneal dissemination. Surgery. 1996;119:437-44.

10. Hall JJ, Loggie BW, Shen P, Beamer S, Douglas Case L, McQuellon R, et al. Cytoreductive surgery with intraperitoneal hyperthermic chemotherapy for advanced gastric cancer. $J$ Gastrointest Surg. 2004;8:454-63.

11. Jacquet $P$, Sugarbaker PH. Clinical research methodologies in diagnosis and staging of patients with peritoneal carcinomatosis. Cancer Treat Res. 1996;82:359-74.
12. Sayag-Beaujard AC, Francois Y, Glehen O, Sadeghi-Looyeh B, Bienvenu J, Panteix G, et al. Intraperitoneal chemo-hyperthermia with mitomycin $\mathrm{C}$ for gastric cancer patients with peritoneal carcinomatosis. Anticancer Res. 1999;19:1375-82.

13. Cunningham D, Allum WH, Stenning SP, Thompson JN, Van de Velde CJ, Nicolson M, et al. Perioperative chemotherapy versus surgery alone for resectable gastroesophageal cancer. $N$ Engl J Med. 2006;355:11-20.

14. Smeenk RM, Verwaal VJ, Zoetmulder FA. Learning curve of combined modality treatment in peritoneal surface disease. $\mathrm{Br}$ J Surg. 2007;94:1408-14.

15. Yan TD, Links M, Fransi S, Jacques T, Black D, Saunders V, et al. Learning curve for cytoreductive surgery and perioperative intraperitoneal chemotherapy for peritoneal surface malignancya journey to becoming a Nationally Funded Peritonectomy Center. Ann Surg Oncol. 2007;14:2270-80.

16. Yan TD, Black D, Sugarbaker PH, Zhu J, Yonemura Y, Petrou G, et al. A systematic review and meta-analysis of the randomized controlled trials on adjuvant intraperitoneal chemotherapy for resectable gastric cancer. Ann Surg Oncol. 2007;14:2702-13. 